\title{
A Nonlinear Optical Waveguide of Poly(1,9-decadiyne)
}

\author{
Haruki Okawa, Motoyoshi SekiYa, Junichiro Osawa, Tatsuo Wada, \\ Akira Yamada, Hiroyuki Sasabe, and Toshiyuki UrYU* \\ Frontier Research Program, RIKEN (The Institute of Physical and Chemical \\ Research) Hirosawa 2-1, Wako, Saitama 351-01, Japan \\ * Institute of Industrial Science, The University of Tokyo, \\ Roppongi 7-22-1, Minato-ku, Tokyo 106, Japan
}

(Received August 11, 1990)

\begin{abstract}
The nonlinear optical properties of poly(1,9-decadiyne) (PDD), which has a diacetylene group in the main chain, were investigated. Using as-prepared films of PDD, no third harmonic generation (THG) was observed. However, after thermal treatment of PDD films, THG appeared and the third order susceptibility, $\chi^{(3)}$, was determined to be $1.2 \times 10^{-12}$ esu. These heat-treated films were very clear and had no absorption between 600 and $2500 \mathrm{~nm}$. A slab waveguide of polymer thin film was formed on a quartz substrate and its light propagation properties was investigated.
\end{abstract}

KEY WORDS Polydiyne / Polydiacetylene / Thermal treatment / Nonlinear Optical Property / Optical Waveguide / Light Propagation / Third Harmonic Generation /

There have been many investigations of organic nonlinear optical (NLO) materials. ${ }^{1}$ Compared with inorganic ones, they have advantages such as a large susceptibility, fast response, and high optical damage threshold. Second order nonlinearity requires noncentrosymmetry in macroscopic structures of materials. Therefore, not only the molecular design for large hyperpolarizabilities but the crystal engineering for the control of molecular aggregates is very important. On the other hand, third order nonlinear effects can be observed in materials of any macroscopic structure $^{2}$ and there is no limitation to the material design. The third order nonlinear active organic materials have the potential to be applied to optical devices such as optical memories, optical logical devices, and all optical computers. $^{3}$

In general, the third order susceptibility $\chi^{(3)}$ is intrinsically small, so that to get a large NLO effect, a long interaction length of light with the material and/or high intensity of light is required. In an optical waveguide, these requirements can be satisfied and hence the waveguide structure is suitable to an optical integrated circuit. ${ }^{4}$ Waveguide materials must have the following properties: (1) absence of absorption at the wavelength of the guided light, (2) absence of scattering of the light, (3) good processability for waveguide fabrication, and (4) adequate mechanical performance. There have been a lot of investigations of third order nonlinear organic materials, especially on $\pi$-electron conjugated systems such as phthalocyanine ${ }^{5}$ polyacetylene, ${ }^{6}$ and polydiacetylene, ${ }^{7}$ which have high $\chi^{(3)}$ values of $10^{-10}$ esu. However, these materials are still far from the waveguide application because of their poor processability.

Compounds with diacetylene groups can be polymerized, by irradiation with light or $\gamma$-rays or by heat treatment, to conjugated polymers. These polymers have interesting properties for optoelectronic devices and exhibit a large third order optical nonlinear effect due to long 
$\pi$-conjugation along the main chain. On the other hand, polymers having diacetylene groups in the main chain can be changed into conjugated cross-linked polymers, so that these polymers are applicable for photoresists. Therefore these cross-linked polymers are expected to behave as a conjugated polydiacetylene. ${ }^{8)}$ In this report, poly(1,9-decadiyne) (PDD), whch contains a diacetylene group in its main chain, was prepared by oxidative coupling polymerization. We will discuss the third order nonlinear effect of the cross-linked PDD polymer estimated from the third harmonic generation measurement, and the light propagation in the slab waveguide of the polymer.

\section{EXPERIMENTAL}

\section{Material}

Poly(1,9-decadiyne) (PDD) was prepared by oxidative polymerization of 1,9-decadiyne

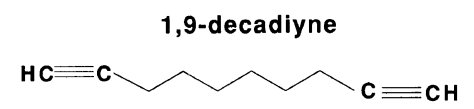

\section{$\mathrm{O}_{2}, \mathrm{CuCl}, \mathrm{N}, \mathrm{N}, \mathrm{N}$ ',N'-tetramethylethylenediamine}

Pyridine, chlorobenzene: $60^{\circ} \mathrm{C}, 5 \mathrm{~h}$

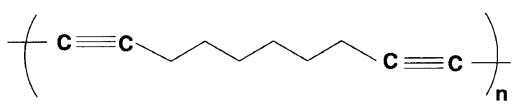

Scheme. Polymerization of poly(1,9-decadiyne). according to the literature. ${ }^{8}$ The chemical structure of the polymer was determined by ${ }^{1} \mathrm{H}$ NMR. No peak was observed at $1.94 \mathrm{ppm}$ due to the methyne proton of the acetylene group which was observed in the monomer spectrum. A film was prepared from a solution of polymer in 1,2-dichloroethane and dried for $24 \mathrm{~h}$ under vacuum. The thickness of the films was determined by using a surface profiler Dektak II. The UV-absorption spectrum of the polymer was recorded by using a Shimadzu UV-3100 spectrometer. Heat-treatment of PDD films was carried out at $150^{\circ} \mathrm{C}$ for $48 \mathrm{~h}$ in a dry oven. These heat-treated PDD films were used for optical measurement.

\section{Measurement}

The third order nonlinear susceptibility, $\chi^{(3)}$, was determined by third harmonic generation (THG) measurement. The THG measuring system is schematically depicted in Figure 1. A Q-switched Nd:YAG laser $(\lambda=1.06 \mu \mathrm{m})$ was used as a light source and converted to light of $\lambda=1.9 \mu \mathrm{m}$ by a hydrogen Raman cell. To remove the effect of air, the sample film was put into a vacuum chamber. ${ }^{2}$

The measuring system for light propagation properties in a slab waveguide of the polymer are shown in Figure 2. A CW Nd: YAG laser and a $\mathrm{He}-\mathrm{Ne}$ laser were used as light sources, and TE modes of the propagating light in a waveguide were excited by the prism coupling method (FD-11 prism, refractive indices

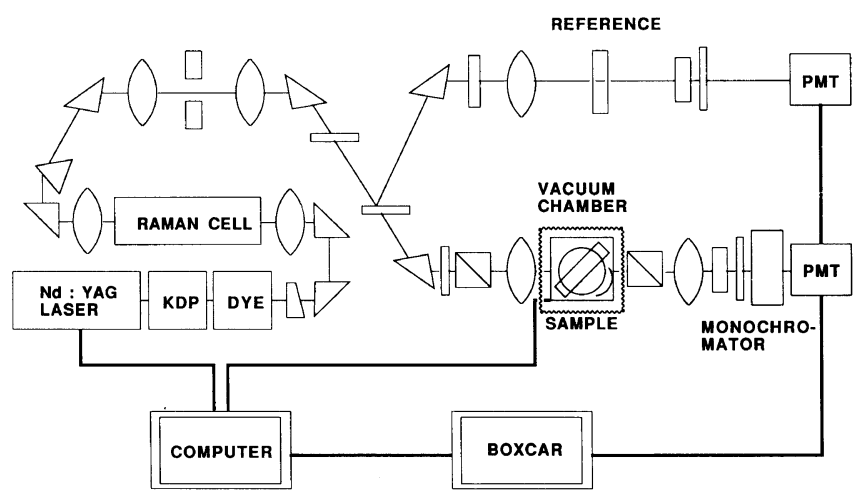

Figure 1. Experimental setup for third harmonic generation. 


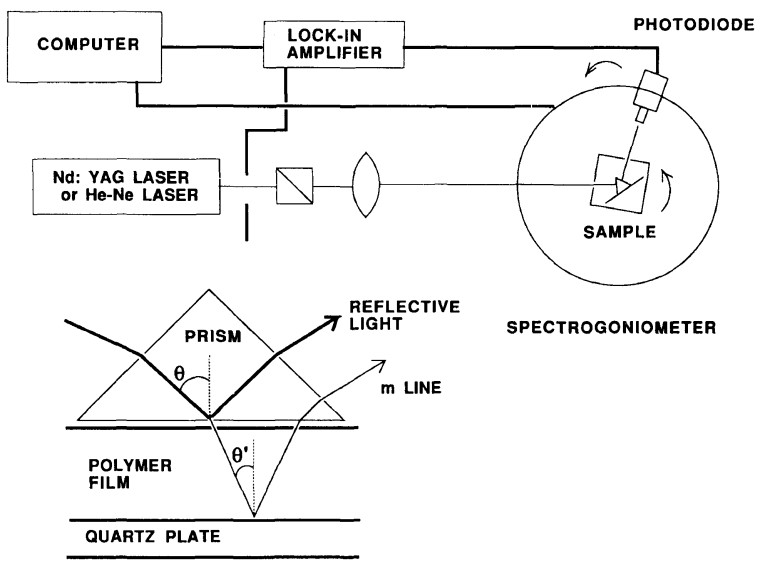

Figure 2. Experimental setup for light propagation measurement.

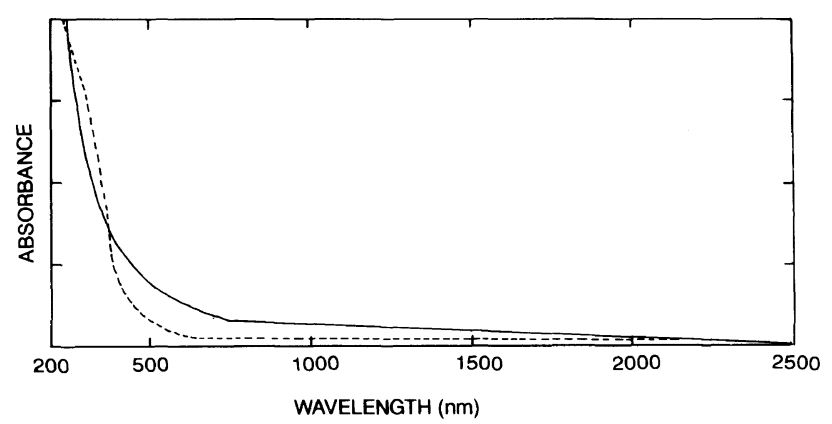

Figure 3. UV-VIS absorption spectra of poly(1,9-decadiyne). (-), as prepared; (-----), heat-treated.

$n=1.7544$ at $\lambda=1.06 \mu \mathrm{m}$ and $n=1.786$ at $\lambda=0.633 \mu \mathrm{m})$. To estimate the light propagation in the waveguide, the reflected light from the bottom of prism was measured by using a photodiode, and the intensity of light was plotted against incident angle.

\section{RESULTS AND DISCUSSION}

UV-absorption spectra of the polymer films are compared in Figure 3. By heat treatment, absorption around $400 \mathrm{~nm}$ increases (dashed curve). This suggests that the diacetylene groups in the polymer main chains reacted to form interchain $\pi$-conjugation. Though the as-prepared film is slightly opaque due to micro-crystals of PDD, it becomes transparent by heat treatment, which is clearly demon- strated as a decrease of the absorption in the longer wavelength region. This result coincides with that of Lando et al. ${ }^{8}$ The absorption spectra of UV-irradiated polymer film exhibits a new peak around $550 \mathrm{~nm}$ due to the conjugation of reacted diacetylene groups (not shown in figure). The film becomes more opaque and brittle after UV-irradiation. A spectrum of the polymer containing a small amount of residual catalyst has a peak at $1900 \mathrm{~nm}$ due to copper ions. The optical measurements were carried out by using the heat-treated films.

A large difference in IR spectra between as-prepared and heat-treated films of PDD is observed (Figure 4). The heat-treated film has a strong absorption peak at $1716 \mathrm{~cm}^{-1}$ due to $\mathrm{C}=\mathrm{C}$ double bonds generated by reaction of 


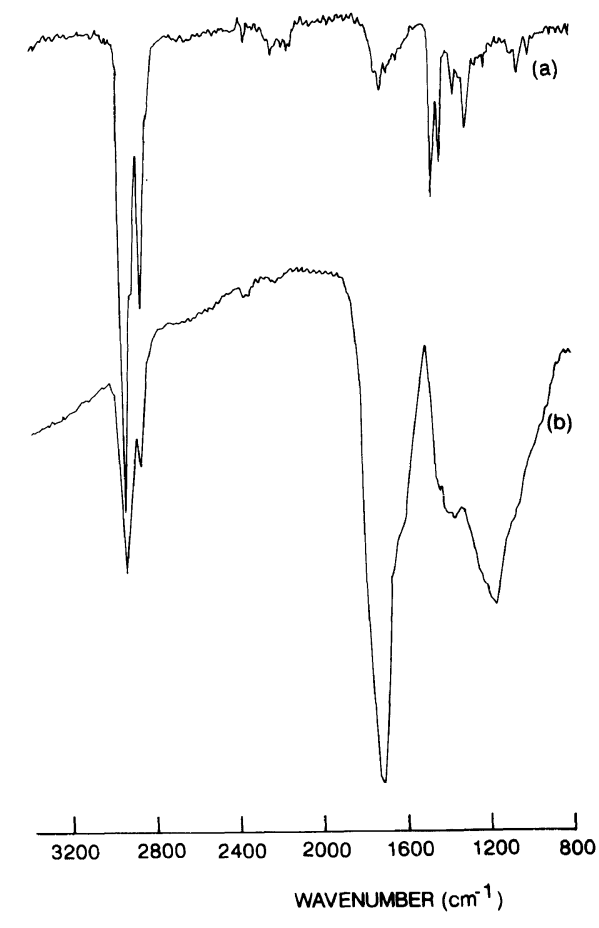

Figure 4. IR absorption spectrum of poly(1,9-decadiyne). (a), as prepared; (b), heat-treated.

diacetylene groups. In principle, symmetric $\mathrm{C}=\mathrm{C}$ double bonds in polydiacetylene cannot be detected by IR-absorption measurement. In this case, however, it is considered that most of diacetylene groups reacted not at 1,4position but at 1,2-position, i.e., the $\mathrm{C}=\mathrm{C}$ double bonds become unsymmetric.

In order to investigate the propagation of light in the slab waveguide of the polymer, the TE mode of the guided wave was excited by a prism coupling method. Figure 5 shows a pattern of output light (He-Ne laser). Bright lines and a spot of high intensity are observed. Because the excited mode in the slab waveguide is guided one-dimensionally, the shape of the output light becomes a line (so called mode-line). The spot is due to the reflection at the prism bottom and observed while the incident angle is changed. The intensity of reflected light ( $\mathrm{Nd}: \mathrm{YAG}$ laser) is plotted against incident angle, as shown in Figure 6. The plot has two minima, indicating that the

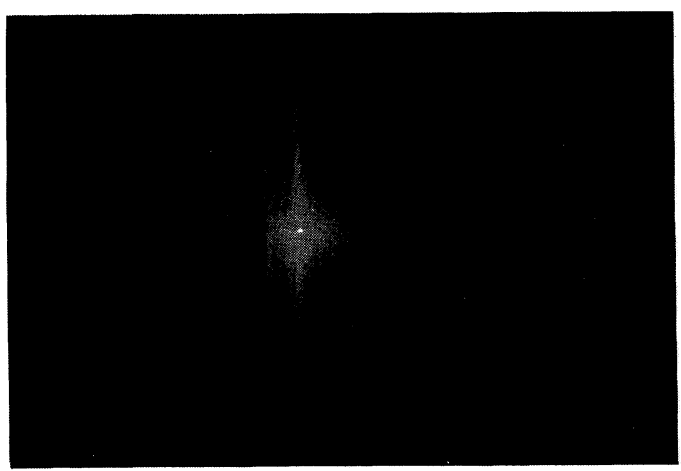

Figure 5. m-Line of $\mathrm{He}-\mathrm{Ne}$ laser from a waveguide of heat-treated poly(1,9-decadiyne).

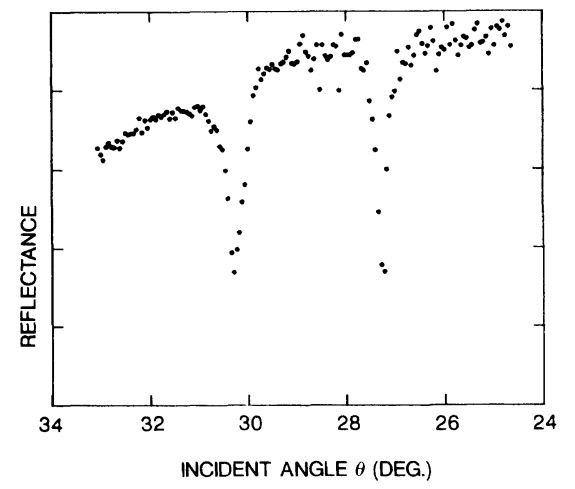

Figure 6. Incident angle dependence of reflected light from prism bottom on the waveguide.

light is introduced into the waveguide and propagates.

A coupling between the light in the prism (refractive index of $n_{\mathrm{p}}$ ) and guided mode in the slab waveguide $\left(n_{\mathrm{f}}\right)$ formed on the substrate $\left(n_{\mathrm{s}}\right)$ takes place along the bottom plane of the prism through a thin gap layer (air) of index $n_{\mathrm{c}}$. The propagation constant $\beta$ of the incident light beam in prism along the waveguide is given by ${ }^{9}$

$$
\beta=n_{\mathrm{p}} k \sin \theta ; \quad k=2 \pi / \lambda
$$

where $\theta$ is the incident angle onto the bottom of the prism. The propagation constant $\beta^{\prime}$ of a mode in a waveguide is expressed as

$$
\beta^{\prime}=k n_{\text {eff }} ; \quad n_{\text {eff }}=n_{\mathrm{f}} \sin \theta^{\prime}
$$




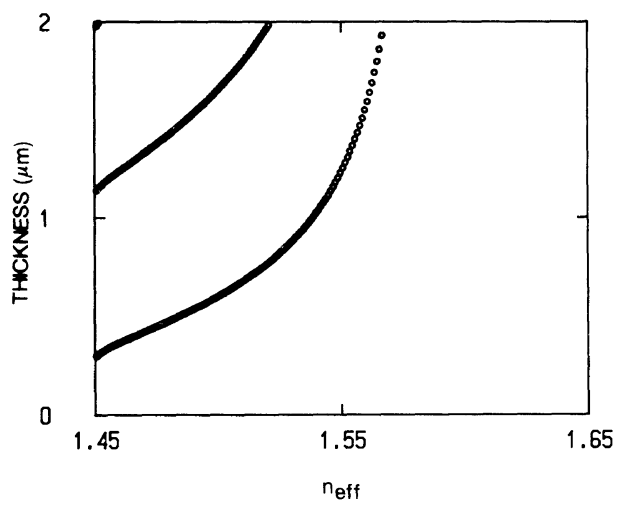

Figure 7. Mode dispersion of a waveguide of poly(1,9decadiyne) for $\mathrm{Nd}$ : YAG laser.

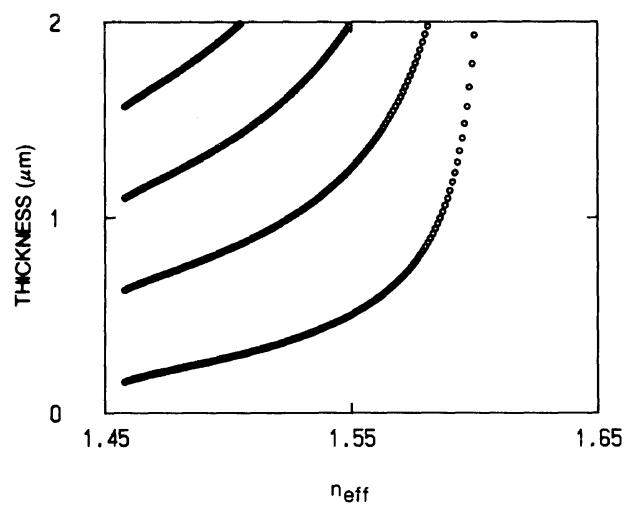

Figure 8. Mode dispersion of a waveguide of poly(1,9decadiyne) for $\mathrm{He}-\mathrm{Ne}$ laser.

where $\theta^{\prime}$ is the total reflection angle. When $\beta$ becomes equal to $\beta^{\prime}$, a guided mode is excited.

From the field equation for TE mode, the wave number in the $x$ direction $k_{x}$ can be derived in the form of eigen value equation,

$$
k_{x} T=(m+1) \pi-\tan ^{-1}\left(r_{\mathrm{s}} / k_{x}\right)-\tan ^{-1}\left(r_{\mathrm{c}} / k_{x}\right)
$$

where $k_{x}=k\left(n_{\mathrm{f}}^{2}-n_{\text {eff }}^{2}\right)^{1 / 2}, r_{\mathrm{c}}=k\left(n_{\text {eff }}{ }^{2}-n_{\mathrm{c}}{ }^{2}\right)^{1 / 2}$, $r_{\mathrm{s}}=k\left(n_{\mathrm{eff}}{ }^{2}-n_{\mathrm{s}}{ }^{2}\right)^{1 / 2}, T$ is the thickness of the waveguide and $m(=0,1,2, \cdots)$ denotes the mode number.

From the angles at which the reflected light become minimum, the mode dispersion of the polymer waveguide is calculated (Figure 7), and the refractive index for $\mathrm{Nd}: \mathrm{YAG}$ laser $(\lambda=1064 \mathrm{~nm})$ is determined to be 1.586 . By
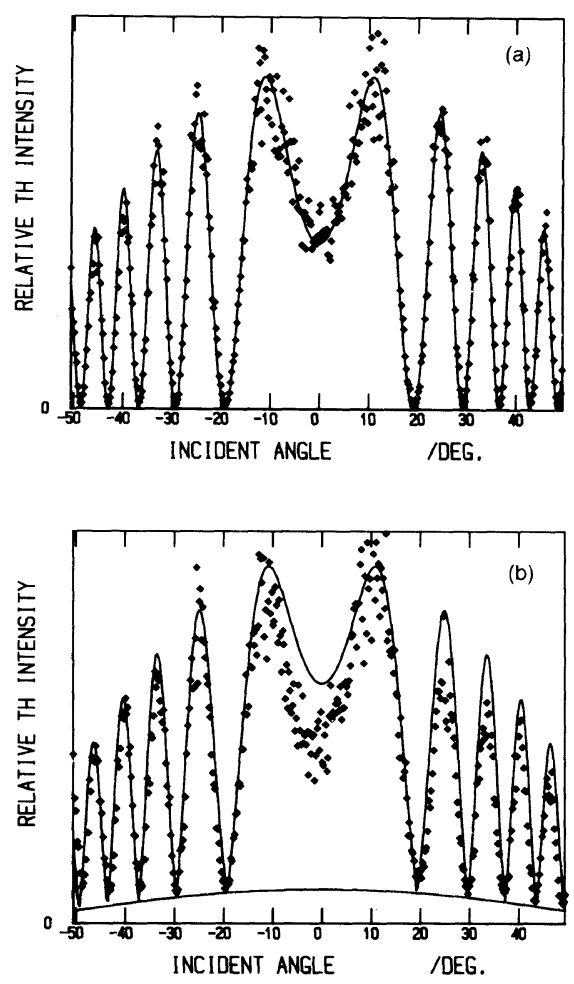

Figure 9. Third harmonic generations from a quartz substrate (a) and a heat-treated film of poly(1,9-decadiyne) on a quartz substrate (b).

using $\mathrm{He}-\mathrm{Ne}$ laser $(\lambda=633 \mathrm{~nm})$ as a light source, the mode dispersion is also obtained (Figure 8) and the refractive index is determined to be 1.607 . These results suggest that the heat-treated PDD film can be used as waveguide because the film neither absorbed nor scattered the lights.

Third order nonlinear susceptibility of the heat-treated film is determined by measuring the intensity of third harmonic generation as a function of incident angle (Maker fringe pattern). Figure 9 shows Makar fringe patterns of the quartz substrate (a) and the polymer film on the substrate (b). The fringe from the quartz substrate has a periodicity, which coorresponds to the successive change of optical pathlengths equal to one, two, or more coherence lengths $\left(l_{c}\right)$ of the quartz substrate, respectively. Also the fringes (b) of the polymer film and the 
substrate shows the periodicity. However, the intenstity of THG did not become zero because of the generation of third harmonic wave in the polymer film. According to Kajzar et al., ${ }^{10}$ these fringe patterns are expressed as

$$
\begin{gathered}
J_{3 \omega}=\frac{256 \pi^{4} J_{3 \omega}^{3}}{C^{2}}\left|\frac{A_{\chi}^{(3)}}{\Delta \varepsilon}\right|^{2} \sin ^{2} \frac{\Delta \Phi}{2} \quad\left(l \sim l_{\mathrm{c}}\right) \\
J_{3 \omega}=\frac{2304 \pi^{6} J_{3 \omega}{ }^{3}}{C^{2}}\left|\frac{A_{\chi}^{(3)}}{n_{\omega}+n_{3 \omega}}\right|^{2}\left(1 / \lambda_{\omega}\right) \\
A=\left(t_{\omega}\right)^{3} T_{3 \omega} \\
t_{\omega}=2 \cos \theta /\left(n_{\omega} \cos \theta_{\omega}+\cos \theta\right) \\
T_{3 \omega}=\left(n_{3 \omega} \cos \theta_{3 \omega}+n_{\omega} \cos \theta_{\omega}\right) /\left(n_{3 \omega}+\cos \theta\right)
\end{gathered}
$$

where the subscripts $\omega$ and $3 \omega$ indicate fundamental and third harmonic waves respectively, $J$ is intensity of wave, $t_{\omega}$ and $T_{3 \omega}$ are Fresnel-like transmission factors, and $\theta, \theta_{\omega}$ and $\theta_{3 \omega}$ are incident angle, light propagation angles of fundamental and harmonic waves, respectively.

To estimate the incident power of fundamental wave, the curve of Figure 9(a) is fitted to eq 3 by introducing parameters of refractive indices, $n_{\omega}=1,450, n_{3 \omega}=1.457$, thickness $l=$ $1 \mathrm{~mm}, \chi^{(3)}=2.8 \times 10^{-14}$ esu for the fused silica substrate. For the polymer film $n_{\omega}$ and $n_{3 \omega}$ are assumed to be 1.607 (at $\lambda=1064 \mathrm{~nm}$ ) and 1.583 (at $\lambda=633 \mathrm{~nm}$ ), respectively, because dispersion of refractive index of the polymer is very little at these wavelength. Since the coherence length $l_{\mathrm{c}}=\lambda / 6\left(n_{\omega}-n_{3 \omega}\right)=13.44 \mu \mathrm{m}$ is less than the sample thickness $l=0.5 \mu \mathrm{m}$, then the curve fitting is carried out by using eq 4 . As a result, the $\chi^{(3)}$ value of the polymer film is calculated as $1.2 \times 10^{-12}$ esu. This value is not so high compared to the values of polydiacetylenes $\left(10^{-10}\right.$ to $\left.10^{-11} \mathrm{esu}\right) .^{11}$ Since the conjugation length of the polymer chain is not large and the absorption edge of the polymer is approximately $500 \mathrm{~nm}$, THG by the light of $\lambda=1.9 \mu \mathrm{m}$ is a non-resonant process. ${ }^{12}$ Therefore, the third order nonlinear susceptibility of the polymer becomes relatively low.

\section{CONCLUSION}

Poly(1,9-decadiyne) (PDD) is prepared by oxidative coupling polymerization. The spincoated film of this polymer was slightly opaque, but by heat treatment the PDD film becomes clear and has no absorption in $\mathrm{He}-\mathrm{Ne}$ laser and Nd: YAG laser regions. The heat treated polymer forms a slab waveguide structure and propagates these laser wavelength. The value of $\chi^{(3)}$ is determined as $1.2 \times 10^{-12}$ esu. This value is relatively low probably because of the short conjugating length of polydiacetylene chains. However, the $\chi^{(3)}$ values of cross-linked polydiynes might be improved through the expansion of the $\pi$-conjugated system. Polydiynes are considered to be promising patternable materials for nonlinear optical waveguides.

\section{REFERENCES}

1. For example: D. J. Williams, Ed., "Nonlinear Optical Properties of Organic and Polymeric Materials," ACS Symposium Series 233, 1983.

2. F. Kajzar and J. Messier, Phys. Rev. A, 32, 2352 (1985).

3. L. Smuelson, A. K. M. Rahman, G. P. Puglia, S. Clough, S. Tripathy, T. Inagaki, X. Q. Yang, T. A. Skotheim, and Y. Okamoto, Proceedings of the International Workshop on "Intelligent Materials," Tsukuba, The Society of Non-Traditional Technology, Tokyo, 1989.

4. G. I. Stegeman and R. H. Stolen, J. Opt. Soc. Am, $B, 6,652$ (1989).

5. T. Wada, Y. Matsuoka, K. Shigehara, A. Yamada, A. F. Garito, and H. Sasabe, in "Photoresponsive Materials," Vol. 12, S. Tazuke, Ed., Proceedings of MRS International Meeting on Advanced Materials, Material Research Society, Pittsburgh, Pennsylvania, 1989, p. 75.

7. C. Sauteret, J. P. Hermann, R. Frey, F. Pradere, J. Ducuing, R. H. Baughman, and R. R. Chance, Phys. Rev., 36, 956 (1976).

8. R. J. Butera, R. P. Grasso, M. K. Thakur, and J. B. Lando, in "Crystallographically Ordered Polymers," D. J. Sandman, Ed., ACS Symposium Series 337, 


\section{A Nonlinear Optical Waveguide of Poly(1,9-decadiyne)}

1986, p. 25.

9. J. H. Harris, R. Shubert, and J. N. Polky, J. Opt. Soc. Am., 60, 1007 (1970).

10. (a) F. Kajzar and J. Messier, Thin Solid Films, 132, 11 (1985); (b), F. Kajzar, J. Messier, and C. Rosilio, J. Appl. Phys., 60, 3040 (1986).
11. (a) C. Grossman, J. R. Heflin, K. Y. Wong, O. Zamani-Khamiri, and A. F. Garito, in "Nonlinear Optical Effects in Organic Polymers," J. Messier, F. Kajzar, P. Prasad, and D. Ulrich, Ed., NATO series 162, 1989, p 61; (b) M. D. Levenson, and N. Bloembergen, Phys. Rev. B, 10, 4447 (1974). 